\title{
Learning curve for robotic-assisted laparoscopic colorectal surgery
}

\author{
Malak B. Bokhari • Chirag B. Patel • \\ Diego I. Ramos-Valadez • Madhu Ragupathi • \\ Eric M. Haas
}

Received: 2 February 2010/Accepted: 19 July 2010/Published online: 24 August 2010

(c) The Author(s) 2010. This article is published with open access at Springerlink.com

\begin{abstract}
Background Robotic-assisted laparoscopic surgery (RALS) is evolving as an important surgical approach in the field of colorectal surgery. We aimed to evaluate the learning curve for RALS procedures involving resections of the rectum and rectosigmoid.

Methods A series of 50 consecutive RALS procedures were performed between August 2008 and September 2009. Data were entered into a retrospective database and later abstracted for analysis. The surgical procedures included abdominoperineal resection (APR), anterior rectosigmoidectomy (AR), low anterior resection (LAR), and rectopexy (RP). Demographic data and intraoperative parameters including docking time (DT), surgeon console time (SCT), and total operative time (OT) were analyzed.
\end{abstract}

The abstract of this study was presented as a poster at the 2010 Society of American Gastrointestinal Endoscopic Surgeons (SAGES) / Canadian Association of General Surgeons (CAGS) 12th World Congress of Endoscopic Surgery in Landover, Maryland.

\section{B. Bokhari}

Department of Surgery, VA Pittsburgh Healthcare System, University Drive C, Pittsburgh, PA 15240, USA

C. B. Patel · D. I. Ramos-Valadez · M. Ragupathi Division of Minimally Invasive Colon and Rectal Surgery, Department of Surgery, University of Texas Medical School at Houston, 7900 Fannin Street, Suite 2700, Houston, TX 77054, USA

E. M. Haas $(\bowtie)$

Division of Minimally Invasive Colon and Rectal Surgery, Department of Surgery, University of Texas Medical School at Houston, Colorectal Surgical Associates Ltd, LLP, 7900 Fannin Street, Suite 2700, Houston, TX 77054, USA e-mail: ehaasmd@houstoncolon.com
The learning curve was evaluated using the cumulative sum (CUSUM) method.

Results The procedures performed for 50 patients $(54 \%$ male) included 25 AR (50\%), 15 LAR (30\%), 6 APR (12\%), and $4 \mathrm{RP}(8 \%)$. The mean age of the patients was 54.4 years, the mean BMI was $27.8 \mathrm{~kg} / \mathrm{m}^{2}$, and the median American Society of Anesthesiologists (ASA) classification was 2. The series had a mean DT of 14 min, a mean SCT of $115.1 \mathrm{~min}$, and a mean OT of $246.1 \mathrm{~min}$. The DT and SCT accounted for $6.3 \%$ and $46.8 \%$ of the OT, respectively. The SCT learning curve was analyzed. The CUSUM $_{\mathrm{SCT}}$ learning curve was best modeled as a parabola, with equation CUSUM $_{S C T}$ in minutes equal to $0.73 \times$ case number ${ }^{2}-31.54 \times$ case number -107.72 $(R=0.93)$. The learning curve consisted of three unique phases: phase 1 (the initial 15 cases), phase 2 (the middle 10 cases), and phase 3 (the subsequent cases). Phase 1 represented the initial learning curve, which spanned 15 cases. The phase 2 plateau represented increased competence with the robotic technology. Phase 3 was achieved after 25 cases and represented the mastery phase in which more challenging cases were managed.

Conclusions The three phases identified with CUSUM analysis of surgeon console time represented characteristic stages of the learning curve for robotic colorectal procedures. The data suggest that the learning phase was achieved after 15 to 25 cases.

Keywords Cumulative sum analysis - Learning curve · Rectosigmoid · Robotic-assisted laparoscopic surgery

Surgical proficiency is improved with ongoing innovations in surgical instrumentation and technology. The acquisition of competency in novel surgical techniques represents a 
"learning curve" for each surgeon. The learning curve, in addition to being a function of the surgeon's understanding of the new technique, technical modifications to the technique, and improvements in support staff and perioperative care [1], is a function of the surgeon's evolving ease with the procedure and performance of more challenging cases.

In 1995, a robotic-assisted laparoscopic system prototype was developed for abdominal procedures [2], and in 2001, Weber et al. [3] performed the first robotic-assisted laparoscopic colectomy for benign disease. Soon afterward, Hashizume and Tsugawa [4] reported three colonic resections for malignant disease using a robotic system. Since then, the robotic-assisted laparoscopic system has gained popularity, with more surgeons using it for colorectal procedures of the pelvis, both benign and malignant. With the adoption of new techniques, it is important to assess the effect on the surgeon's learning curve.

The cumulative sum (CUSUM) technique is a method originally devised for monitoring performance and detecting areas for improvement in the industrial sector. This method was adopted by the medical profession in the 1970s to analyze the learning curve for surgical procedures [5, 6]. Cumulative sum analysis transforms raw data into the running total of data deviations from their group mean, enabling investigators to visualize the data for trends not discernable with other approaches.

Multiple reports on robotic-assisted laparoscopic surgery have been published, but none have evaluated the learning curve in robotic colorectal surgery using CUSUM analysis. We aimed to analyze the learning curve for robotic-assisted laparoscopic rectosigmoid surgery using CUSUM methodology.

\section{Materials and methods}

This study was approved by the institutional review board. Data were abstracted into a retrospective database for analysis. Fifty consecutive robotic-assisted laparoscopic surgery (RALS) procedures were performed between August 2008 and September 2009 by an experienced laparoscopic colorectal surgeon (E.M.H.).

Docking time (DT) was defined as the time required to position the robot and secure the robotic arms to the corresponding port sites. The surgeon console time (SCT) was the actual time the surgeon spent at the robotic console during the procedure, which directly corresponded to the robotic portion of the procedure. The total operative time (OT) spanned the time from the first incision to the final closure. The surgical procedures included abdominoperineal resection (APR), anterior rectosigmoidectomy (AR), low anterior resection (LAR), and rectopexy (RP).
All the procedures were performed in a medial-to-lateral approach, with early identification and ligation of the inferior mesenteric artery and vein when indicated. The left ureter and hypogastric plexus were routinely identified and preserved. When required, the splenic flexure was taken down using conventional laparoscopic technique. The anastomoses were performed intracorporeally with the ECS29 circular stapling device (Endopath ${ }^{\circledR}$ ILS; Ethicon Endo-Surgery, Cincinnati, OH, USA).

Patients who underwent pelvic radiation were routinely diverted with loop ileostomy. Demographic data including patient gender, age, body mass index (BMI), and American Society of Anesthesiologists (ASA) score were tabulated. Intraoperative parameters including DT, SCT, OT, and estimated blood loss (EBL) were analyzed as well as the patient's hospital length of stay (LOS).

Cumulative sum analysis

The CUSUM technique was used for quantitative assessment of the learning curve. The CUSUM is the running total of differences between the individual data points and the mean of all data points. Thus, CUSUM can be performed recursively. The CUSUM technique was used for 48 cases that had SCT data available.

First, the cases were ordered chronologically, from the earliest to the latest date of surgery. The CUSUM ${ }_{\mathrm{SCT}}$ of the first case was the difference between the SCT for the first case and the mean SCT for all the cases $\left(\mu_{\mathrm{SCT}}\right)$. The CUSUM $_{\mathrm{SCT}}$ of the second case was the previous case's CUSUM $_{\mathrm{SCT}}$ added to the difference between the SCT for the second case and $\mu_{\mathrm{SCT}}$. This recursive process continued

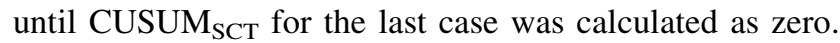
Because no patient deaths occurred in this series, riskadjusted CUSUM (RA-CUSUM) [7, 8] was not performed.

Statistical analysis

Statistical analysis was performed using Intercooled Stata version 9 software (StataCorp LP, College Station, TX, USA). For interphase comparisons, the Wilcoxon rank-sum test was used (alpha after Bonferroni correction $=0.0167$ ). Comparisons also were made between phases 1 and 2 combined (learning curve) and phase 3 (competency) using a two-tailed Student's $t$-test (alpha $=0.05$ ).

\section{Results}

During the study period, 50 patients underwent RALS for rectosigmoid resection. Patient demographics, preoperative diagnoses, surgical procedures, operative characteristics, and postoperative outcomes are summarized in Table 1. 
Table 1 Patient demographics, preoperative diagnoses, surgical procedures, operative

characteristics, and

postoperative outcomes $(n=50$, unless otherwise specified)

ASA American Society of Anesthesiologists, $B M I$ body mass index, $A R$ anterior resection, $L A R$ lower anterior resection, $A P R$

abdominoperineal resection, $R P$ rectopexy, $D T$ docking time, $S C T$ surgeon console time, $E B L$ estimated blood loss, $O T$ total operative time, $L O S$ hospital length of stay

\begin{tabular}{lllll}
\hline Category & Parameter & Mean \pm SD & Range & Median \\
\hline Patient characteristics & Age (years) & $54.4 \pm 13.1$ & $24-82$ & 53.5 \\
& ASA score & $2.3 \pm 0.5$ & $2-4$ & 2 \\
& BMI $\left(\mathrm{kg} / \mathrm{m}^{2}\right)$ & $27.8 \pm 6.3$ & $16-49.4$ & 26.9 \\
& Gender: 27 male $(54 \%), 23$ female $(46 \%)$ & \\
Preoperative & Malignant $(n=22 ; 44 \%)$ & \\
Diagnosis & Diverticulitis $(n=18,36 \%)$ & \\
& Rectal prolapse $(n=5,10 \%)$ & \\
& Endometriosis $(n=2,4 \%)$ & \\
Surgical & Other pathology $(n=3,6 \%)$ & & \\
Procedure & AR $(n=25,50 \%)$ & & \\
& LAR $(n=15,30 \%)$ & & \\
& APR $(n=6,12 \%)$ & & 112.5 \\
Intraoperative parameters & RP $(n=4,8 \%)$ & & \\
& DT (min, $n=48)$ & $14.0 \pm 7.7$ & $6-45$ \\
& SCT (min, $n=48)$ & $115.1 \pm 46.9$ & $40-210$ & \\
& OT (min) & $246.1 \pm 80.7$ & $90-540$ & 240 \\
Short-term postoperative outcome & LOS (days) & $3.5 \pm 2.3$ & $2-16$ & 3 \\
\hline
\end{tabular}

The series comprised 23 females (46\%) and 27 males (54\%) with a mean age of 54.4 years, a mean BMI of $27.8 \mathrm{~kg} / \mathrm{m}^{2}$, and a median ASA of 2. Surgery was performed for malignant disease in 22 cases (44\%) and benign disease in 28 cases $(56 \%)$. Of the 50 patients, $25(50 \%)$ underwent AR, 15 had LAR (30\%), 6 had APR (12\%), and $4(8 \%)$ had RP. The majority of ARs (72\%) were performed for diverticular disease, whereas the majority of LARs $(66.7 \%)$ were performed for malignant disease. All 6 APRs were performed for malignant disease, including 5 for anal cancer. The mean DT was 14 min (range, 6-45 min), the mean SCT was $115.1 \mathrm{~min}$ (range, 40-210 $\mathrm{min}$ ), and the mean OT was $246.1 \mathrm{~min}$ (range, $90-540 \mathrm{~min}$ ). The DT and SCT accounted for $6.3 \%$ and $46.8 \%$ of the OT, respectively.

The raw SCT times were plotted in chronological case order (Fig. 1A). The CUSUM SCT $_{\text {learning curve was best }}$ modeled as a second-order polynomial (parabola) with equation CUSUM $_{\mathrm{SCT}}$ in minutes equal to $0.73 \times$ case number $^{2}-31.54 \times$ case number -107.72 , which had a

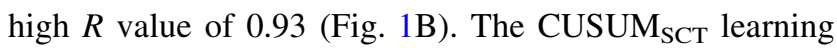
curve was observed to consist of three unique phases: phase 1 (the initial 15 cases), phase 2 (the middle 10 cases), and phase 3 (the final 23 cases) (Fig. 2).

Comparisons of various parameters between the three

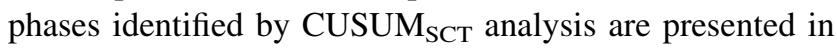
Table 2. Age, BMI, ASA, and previous surgical history did not differ significantly among the three phases. The proportion of cases involving performance of an anastomosis (AR and LAR) differed significantly between phase 3 and phases 1 and 2 combined $(p<0.008)$.
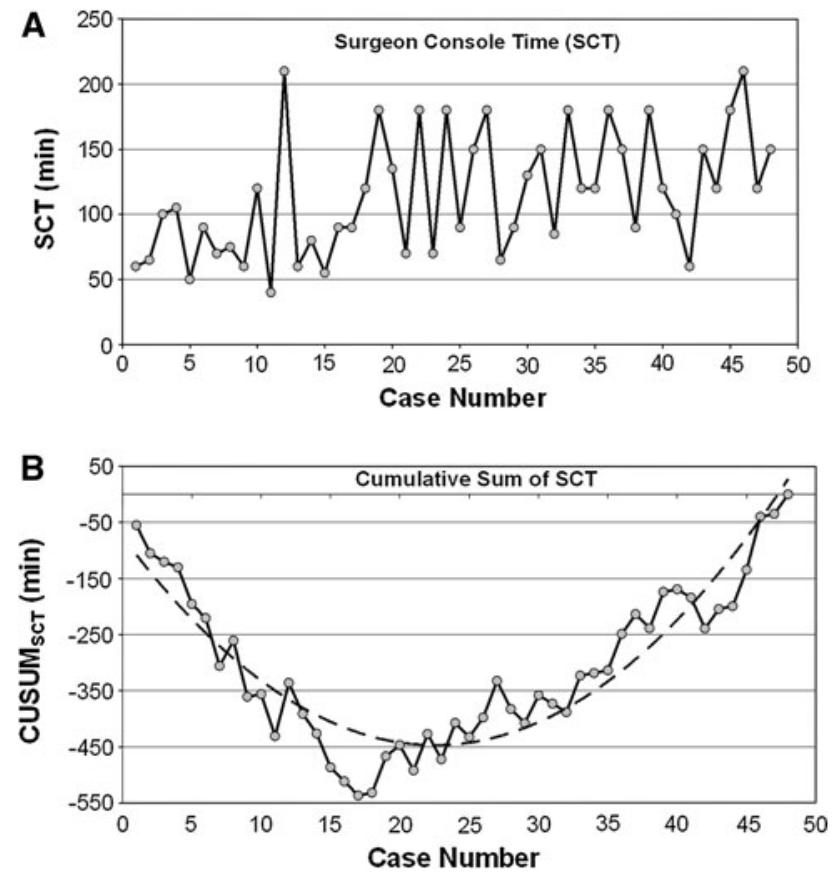

Fig. 1 Surgeon console time (SCT). A SCT plotted against case number. B Cumulative sum (CUSUM) $)_{\mathrm{SCT}}$ plotted against case number (solid line). The dashed line represents the curve of best fit for the plot (a second-order polynomial with equation CUSUM $_{\mathrm{SCT}}=0.73 \times$ case number $^{2}-31.54 \times$ case number $-107.72(R=0.93)$

Interphase comparisons of intraoperative parameters (operative times and EBL) and short-term postoperative outcome (LOS) are presented in Table 3. A significant reduction in DT and EBL was observed between phases 1 


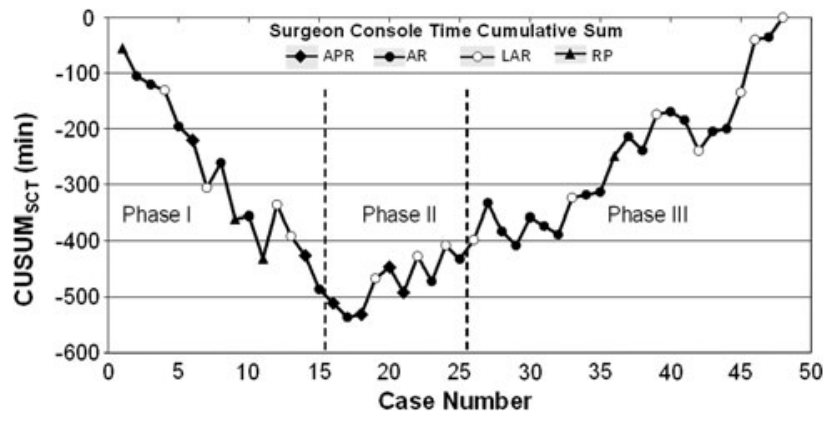

Fig. 2 Three phases of the surgeon console time (SCT) in terms of the cumulative sum (CUSUM) learning curve. The solid diamond represents abdominoperineal resection (APR), and the solid circle represents anterior resection (AR). The open circle represents low anterior resection (LAR), and the solid triangle represents rectopexy (RP)

and 2 compared with phase $3(p<0.0002$ and $<0.03$, respectively). The mean SCT for the first 15 cases (phase 1: $82.7 \pm 41.6 \mathrm{~min})$ was significantly shorter than for the last 23 cases (phase 3: $133.9 \pm 40.6 \mathrm{~min})(p<0.001)$. Figure 3 displays the lines of best fit for the three phases of the SCT learning curve.

\section{Discussion}

The learning curve is a graphic representation of the temporal relationship between the surgeon's mastery of a specifically assigned task and the chronological number of cases performed. The CUSUM technique is a method adopted by the medical profession in the 1970s to analyze the learning curve for surgical procedures $[5,6]$. We used the CUSUM method to investigate the learning curve for robotic-assisted (da Vinci ${ }^{\circledR}$ System, Intuitive Surgical, Inc., Sunnyvale, CA) rectosigmoid and rectal surgery in the pelvis for benign and malignant colorectal disease. The focus of this study was on investigating surgeon console time as a surrogate marker for operative competency by dividing operative time into phases shown to correlate with process components of surgeon learning.

Publications investigating the learning curve in robotic surgery have performed their analysis based on chronological cases split into predefined segments (e.g., quartiles), with univariate analysis performed to compare means across segments. For instance, Bell et al. [9] reviewed operative times for 100 consecutive robotic-assisted hysterectomies by dividing the series into 20 -case quintiles.

Table 2 Interphase comparisons of patient characteristics and other parameters (mean \pm standard deviation)

\begin{tabular}{|c|c|c|c|c|}
\hline Characteristic & $\begin{array}{l}\text { Phase } 1 \\
(n=15)\end{array}$ & $\begin{array}{l}\text { Phase } 2 \\
(n=10)\end{array}$ & $\begin{array}{l}\text { Phase } 3 \\
(n=23)\end{array}$ & $\begin{array}{l}\text { Phases } 1 \text { and } 2 \text { vs } \\
\text { Phase } 3 \text { ( } p \text {-value) }\end{array}$ \\
\hline Age (years) & $51.7 \pm 12.5$ & $59.0 \pm 8.3$ & $53.2 \pm 14.7$ & NS \\
\hline BMI $\left(\mathrm{kg} / \mathrm{m}^{2}\right)$ & $26.8 \pm 8.0$ & $27.2 \pm 4.6$ & $28.7 \pm 5.7$ & NS \\
\hline ASA & $2.5 \pm 0.6$ & $2.4 \pm 0.4$ & $2.2 \pm 0.4$ & NS \\
\hline Female/male & $10: 5$ & $4: 6$ & $8: 15$ & NS \\
\hline Malignant disease (\%) & $4(26.7)$ & $7(70)$ & $9(39.1)$ & NS \\
\hline APR (\%) & $2(13.3)$ & $4(40)$ & $0(0)$ & $<0.013^{\mathrm{a}}$ \\
\hline $\mathrm{RP}(\%)$ & $3(20)$ & $0(0)$ & $1(4.3)$ & NS \\
\hline LAR and AR (anastomosis) (\%) & $10(66.7)$ & $6(60)$ & $22(95.7)$ & $<0.008^{\mathrm{a}}$ \\
\hline
\end{tabular}

$A S A$ American Society of Anesthesiologists, $A P R$ abdominoperineal resection, $A R$ anterior resection, $B M I$ body mass index, $L A R$ low anterior resection, $N S$ not statistically significant, $R P$ rectal prolapse

a Statistically significant

Table 3 Interphase comparisons of intraoperative parameters and short-term outcome

\begin{tabular}{lcccc}
\hline Operative time & $\begin{array}{l}\text { Phase 1 } \\
(n=15)\end{array}$ & $\begin{array}{l}\text { Phase 2 } \\
(n=10)\end{array}$ & $\begin{array}{l}\text { Phase 3 } \\
(n=23)\end{array}$ & $\begin{array}{l}\text { Phases 1 and 2 vs } \\
\text { Phase 3 }(p \text {-value })\end{array}$ \\
\hline DT (min) & $19.5 \pm 9.7$ & $15.3 \pm 6.9$ & $10.0 \pm 3.0$ & $<0.0002^{\mathrm{a}}$ \\
SCT (min) & $82.7 \pm 41.6$ & $120.5 \pm 45.6$ & $133.9 \pm 40.6$ & $<0.045^{\mathrm{a}}$ \\
OT (min) & $214.0 \pm 74.2$ & $238.0 \pm 71.0$ & $269.8 \pm 84.2$ & $<0.048^{\mathrm{a}}$ \\
EBL (ml) & $129.7 \pm 70.7$ & $112.5 \pm 39.5$ & $85.9 \pm 49.3$ & $<0.03^{\mathrm{a}}$ \\
LOS (days) & $3.8 \pm 3.6$ & $3.8 \pm 2.1$ & $3.1 \pm 0.9$ & NS \\
\hline
\end{tabular}

$D T$ robotic docking time, $E B L$ estimated blood loss, $L O S$ hospital length of stay, $N S$ not statistically significant, $O T$ total operative time, $S C T$ surgeon console time

a Statistically significant 

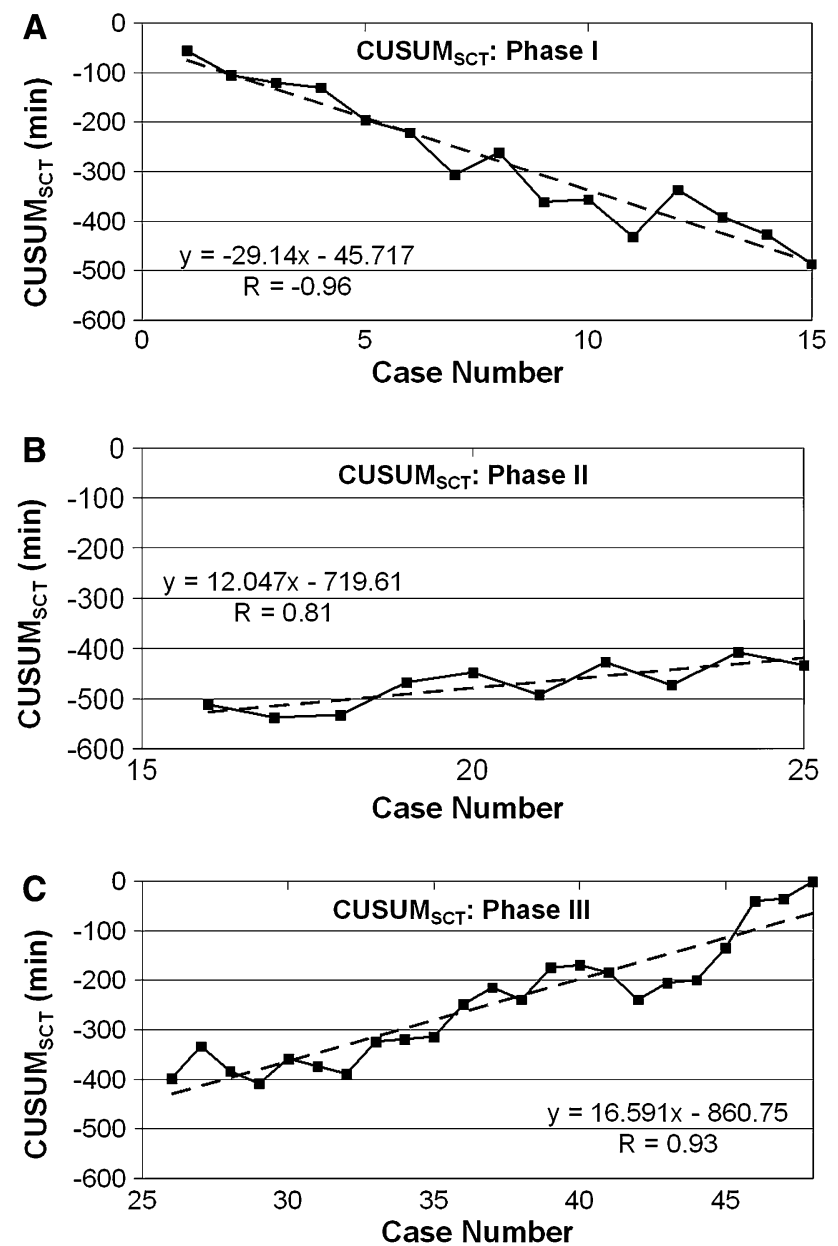

Fig. 3 Lines of best fit for each phase of the cumulative sum $(\text { CUSUM })_{\mathrm{SCT}}$ learning curve. A Phase 1 represents the initial learning curve. B Phase 2 represents the accumulation of additional experience. C Phase 3 represents increasing surgeon competence

Operative times and complication rates decreased over the study period, and maximum improvement was observed after the first quintile. A similarly designed study was reported by Tsao et al. [10], who divided their first 100 cases of robotic-assisted laparoscopic prostatectomy into 25-case quartiles. Total operative time and EBL decreased over the study period, but the most significant improvement in OT and EBL was observed after 25 cases and 50 cases, respectively.

Additionally, CUSUM analysis has been used to analyze the learning curve in conventional laparoscopic colorectal surgery [11]. Tekkis et al. [1, 12] reported two such series. One of the two studies compared right and left colectomy [12]. The analysis demonstrated a learning curve of 55 cases for right-sided and 62 cases for left-sided colectomy. The median operative time declined with operative experience. The readmission rate and the postoperative complications were not dependent on operative experience. The second study investigated RA-CUSUM in ileal-pouch anal anastomosis [1]. Pouch failure was the primary end-point, and the trainee staff showed an improvement in pouch failure rate after 23 cases. To our knowledge, however, a CUSUM-based approach for the analysis of the robotic learning curve in colorectal cases has yet to be reported.

Our study used the CUSUM method to investigate the learning curve in RALS. The study report describes the experience at a single institution by the same surgeon (E.M.H.), who has been performing minimally invasive colorectal surgery since 2002. The SCT was analyzed in depth because it represents the surgeon's time at the robotic console and, based on pairwise correlation analysis, it correlated strongly with OT $(R=0.76 ; p<0.00001)$.

We chose CUSUM analysis because meaningful conclusions cannot be drawn from raw data plotted by chronological cases (Fig. 1A). The CUSUM SCT $_{\text {graph (Fig. 1B) shows }}$ the variance from the mean on a case-by-case basis, yielding a parabolic curve with three distinct phases from which correlates of the learning curve can be assessed (Fig. 2). The large magnitude of the $R$ values $(-0.96,0.81$, and 0.93 , respectively) in Fig. 3 for the line of best fit in each phase indicates the unique components of the surgeon's learning curve for RALS colorectal procedures. The negative slope in phase 1 indicates shorter SCTs during this learning curve phase (lower with respect to the mean SCT over all cases). The positive slope in phases 2 and 3 indicates longer SCTs (greater with respect to the mean SCT over all cases), which are necessary for the performance of more complicated cases taken on with increased surgeon competence.

We believe the learning curve entails the surgeon's mastery of three important and unique facets of roboticassisted technology: (1) overcoming the loss of tensile and tactile feedback by recognizing visual cues with regard to tension and manipulation of the tissues, (2) conceptualizing the spatial relationships of robotic instruments outside the active field of view to manipulate and reposition safely without direct visualization, and (3) mentally visualizing the spatial relationships of the robotic arms and cart (and blinded to these external movements) while operating at the console, thereby minimizing external clashing and optimizing maneuverability and range of motion. To facilitate the acquisition of such unique facets in a safe and stepwise fashion, we believe it is important for a surgeon to acquire expert laparoscopic skills before transitioning to the robotic approach.

Phase 1 represents the initial learning curve phase, found to include 15 cases. An additional 10 cases comprises phase 2 , which represents the accumulation of additional experience once the initial learning curve has been achieved. In our series, we found that the intraoperative complication rate was not dependent on operative experience. However, two recognized thermal injuries occurred during phase 1 , the learning curve. The phase 2 
plateau represents increased competence with the robotic technology. Our results showed the expected decline in SCT (phase 1) followed by a plateau (phase 2), as seen in typical learning curve studies.

The increased operative time in the post-learning period (phase 3) was attributed to a greater proportion of more technically challenging cases in phase 3 as well as to an increased fraction of morbidly obese patients and those with low pelvic malignancies. For instance, significantly more procedures required anastomosis (LAR and AR) in phase 3 than in phases 1 and 2, in which a greater proportion of APRs and RPs were performed. Furthermore, the male-to-female ratio was reversed between phases 1 and 3 . Specifically, phase 1 had twice as many females as males, whereas phase 3 had 1.9 times as many males as females. The male pelvis is considered to be more narrow and confined than the female pelvis, thereby limiting visibility and affecting outcomes in colorectal procedures of the deep pelvis [13]. Despite this trend of a longer OT, however, EBL was significantly diminished in phase 3, and the LOS was not significantly longer. This was also found in a study in which a longer OT during laparoscopic sigmoid colectomy was not associated with an increased complication rate or a longer LOS [14].

This study, using CUSUM analysis, identified three unique phases of the learning curve in the field of roboticassisted laparoscopic colorectal surgery. The data suggest that after a learning curve phase of 15 to 25 cases, the surgeon may achieve a higher level of competence and consider offering this approach to patients presenting with more complicated cases. Overcoming the learning curve involves mastery of the visual cues as well as both the internal and external spatial relationships unique to the robotic approach.

Disclosures Malak B. Bokhari, Chirag B. Patel, Diego I. RamosValadez, Madhu Ragupathi, and Eric M. Haas have no conflicts of interest or financial ties to disclose.

Open Access This article is distributed under the terms of the Creative Commons Attribution Noncommercial License which permits any noncommercial use, distribution, and reproduction in any medium, provided the original author(s) and source are credited.

\section{References}

1. Tekkis PP, Fazio VW, Lavery IC, Remzi FH, Senagore AJ, Wu JS, Strong SA, Poloneicki JD, Hull TL, Church JM (2005) Evaluation of the learning curve in ileal pouch-anal anastomosis surgery. Ann Surg 241:262-268

2. Taylor RH, Funda J, Eldridge B, Gomory S, Gruben K, LaRose D, Talamini M, Kavoussi L, Anderson J (1995) A telerobotic assistant for laparoscopic surgery. IEEE Eng Med Biol 14: 279-288

3. Weber PA, Merola S, Wasielewski A, Ballantyne GH (2002) Telerobotic-assisted laparoscopic right and sigmoid colectomies for benign disease. Dis Colon Rectum 45:1689-1694 discussion $1695-1686$

4. Hashizume M, Tsugawa K (2004) Robotic surgery and cancer: the present state, problems, and future vision. Jpn J Clin Oncol 34:227-237

5. Chaput de Saintonge DM, Vere DW (1974) Why don't doctors use CUSUMs? Lancet 1:120-121

6. Wohl H (1977) The CUSUM plot: its utility in the analysis of clinical data. N Engl J Med 296:1044-1045

7. Biswas P, Kalbfleisch JD (2008) A risk-adjusted CUSUM in continuous time based on the Cox model. Stat Med 27: 3382-3406

8. Steiner SH, Cook RJ, Farewell VT, Treasure T (2000) Monitoring surgical performance using risk-adjusted cumulative sum charts. Biostatistics 1:441-452

9. Bell MC, Torgerson JL, Kreaden U (2009) The first 100 da Vinci hysterectomies: an analysis of the learning curve for a single surgeon. S D Med 62(91):93-95

10. Tsao AK, Smaldone MD, Averch TD, Jackman SV (2009) Robotassisted laparoscopic prostatectomy: the first 100 patientsimproving patient safety and outcomes. J Endourol 23:481-484

11. Delaney CP, Lynch AC, Senagore AJ, Fazio VW (2003) Comparison of robotically performed and traditional laparoscopic colorectal surgery. Dis Colon Rectum 46:1633-1639

12. Tekkis PP, Senagore AJ, Delaney CP, Fazio VW (2005) Evaluation of the learning curve in laparoscopic colorectal surgery: comparison of right-sided and left-sided resections. Ann Surg 242:83-91

13. Seike K, Koda K, Oda K, Kosugi C, Shimizu K, Miyazaki M (2009) Gender differences in pelvic anatomy and effects on rectal cancer surgery. Hepatogastroenterology 56:111-115

14. Scheer A, Martel G, Moloo H, Sabri E, Poulin EC, Mamazza J, Boushey RP (2009) Laparoscopic colon surgery: does operative time matter? Dis Colon Rectum 52:1746-1752 